\title{
The function of engrailed and the specification of Drosophila wing pattern
}

\author{
Isabel Guillén ${ }^{1, *}$, Jose L. Mullor*, Javier Capdevila ${ }^{2}$, Ernesto Sánchez-Herrero, Ginés Morata \\ and Isabel Guerrero' \\ Centro de Biología Molecular Severo Ochoa (CSIC-UAM), Universidad Autónoma de Madrid, Cantoblanco, 28049 Madrid, Spain \\ *The first two authors contributed equally to this work. \\ †Author for correspondence \\ ${ }^{1}$ Present address: Centro de Ingeniená Genética y Biótecnología, PO Box 6162, La Habana 6, Cuba \\ 2Present address: Department of Genetics, Harvard Medical School, Boston, Massachusetts 02115, USA
}

\section{SUMMARY}

The adult Drosophila wing (as the other appendages) is subdivided into anterior and posterior compartments that exhibit characteristic patterns. The engrailed (en) gene has been proposed to be paramount in the specification of the posterior compartment identity. Here, we explore the adult en function by targeting its expression in different regions of the wing disc. In the anterior compartment, ectopic en expression gives rise to the substitution of anterior structures by posterior ones, thus demonstrating its role in specification of posterior patterns. The en-expressing cells in the anterior compartment also induce high levels of the hedgehog (hh) and decapentaplegic (dpp) gene products, which results in local duplications of anterior patterns.
Besides, $h h$ is able to activate $e n$ and the engrailed-related gene invected (inv) in this compartment. In the posterior compartment we find that elevated levels of en product result in partial inactivation of the endogenous $e n$ and $i n v$ genes, indicating the existence of a negative autoregulatory mechanism. We propose that $e n$ has a dual role: a general one for patterning of the appendage, achieved through the activation of secreted proteins like $h \boldsymbol{h}$ and $\boldsymbol{d p p}$, and a more specific one, determining posterior identity, in which the inv gene may be implicated.

Key words: Drosophila, wing disc, compartment, engrailed, hedgehog, invected

\section{INTRODUCTION}

One basic feature of the organization of Drosophila is the subdivision of the body into metameres called parasegments, which appear to define the limits of patterning processes in the embryo (see Lawrence, 1992, for a general overview). The engrailed (en) gene plays an essential role both in the establishment of the parasegmental subdivision of the embryo (Kornberg, 1981a; Ingham et al., 1985) and in the maintenance of parasegmental borders in adult appendages (Morata and Lawrence, 1975; Lawrence and Morata, 1976).

The function of en was originally studied in the adult structures (Garcia-Bellido and Santamaria, 1972; Morata and Lawrence, 1975; Lawrence and Morata, 1976), especially the wing, where anterior and posterior compartments were first found (Garcia-Bellido et al., 1973). Subsequent work indicated that it performs a similar role in other structures such as the antenna and the abdominal histoblasts (Morata and Lawrence, 1979; Kornberg, 1981b; Morata et al. 1983). It was found that the maintenance of the anteroposterior compartment border in wing cells requires normal en function; wings of $e n^{1}$ mutants failed to fix the lineage boundary that normally separates the anterior and posterior compartments (Morata and Lawrence, 1975). Furthermore, mosaic analysis demonstrated that this requirement is specific to posterior compartment cells. In addition to the lack of a proper anteroposterior border, the posterior wing compartment of the $e n^{1}$ (or $e n^{1} / e n^{2}$ ) mutants shows a vein and bristle pattern resembling that of the anterior compartment (Garcia-Bellido and Santamaria, 1972; Lawrence and Morata, 1976). These observations led to the proposal (Lawrence and Morata, 1976) that $e n$ is active in the posterior compartments where it specifies "posterior" identity, be this in the wing, leg or antenna. The implication of this idea is that the critical difference between an anterior and a posterior compartment is provided by the en product; its presence would determine posterior patterns while its absence would specify anterior ones. It is implicit in this hypothesis that, for example in the wing, the complete loss of en function will produce a complete transformation of the posterior compartment into the anterior one, while ectopic expression in the anterior compartment would transform it into a posterior compartment. Studies on the expression of the en product (DiNardo et al., 1985; Fjose et al., 1985; Brower, 1986; Kornberg et al., 1985) supported some features of the model; it was found that en encodes a DNA binding protein restricted to the posterior compartments of embryonic and adult segments, although there is a late en expression in some cells of the anterior compartment close to the border (Blair, 1992).

However, other results indicated a genetic and functional complexity not foreseen by the model. One complication is that lethal (presumably null) en mutations do not show a complete transformation of posterior into anterior patterns (Lawrence and Struhl, 1982; Gubb, 1985). Besides, mutant en clones sometimes produce extra growth in the wing blade (Lawrence 
and Morata, 1976; Lawrence and Struhl, 1982). The situation is further complicated by the finding (Coleman et al., 1987) that there are two similar genes arranged in tandem, en and invected (inv), which encode products of similar sequence. While the existence of inv may help to explain the incomplete transformation of mutant cells for lethal en alleles (Lawrence and Struhl, 1982), assuming en and inv may encode partially redundant functions, the fact is that no individual inv mutation has so far been isolated and so its role, if any, remains unknown. Only very recently a chromosome deficient for both en and inv has been made and mosaic analysis using this chromosome has shown that in the posterior wing margin, doubly mutant cells show a complete transformation of posterior into anterior pattern (Hidalgo, 1994).

Although its expression is restricted to posterior compartments, an indirect participation of $e n$ in anterior adult patterns has been suggested. Recent experiments have shown that $h h$, a posterior specific gene controlled by en (Tabata et al., 1992; Lee et al., 1992), activates decapentaplegic (dpp), an anterior gene involved in the patterning of anterior and posterior compartments (Tabata and Kornberg, 1994; Basler and Struhl, 1994; Capdevila and Guerrero, 1994). These studies have led to a better understanding of the role of $e n$ in the patterning of anterior compartments, but it is unknown if en is sufficient to provide posterior identity in imaginal discs.

Some of the problems concerning the function of $e n$ can be approached by studying the morphogenetic consequences of its ectopic expression in anterior compartments. We have made use of the GAL4 method (Brand and Perrimon, 1993) to express the en product ectopically in the anterior wing disc compartment. We find that it causes a transformation toward posterior patterns in the region containing the product, but also a duplication of anterior patterns in the adjacent region. The anterior to posterior transformation is accompanied by activation of the inv gene, while the anterior duplications correlate with high ectopic levels of $d p p$. The hh product appears to be associated with these two roles, inducing expression of $e n$, inv and $d p p$. We propose that en has a dual function: (1) a non-cell autonomous function necessary for the proliferation and patterning of the entire appendage, achieved through the control of $d p p$ activity and (2) a cell autonomous function responsible for the determination of posterior compartment identity, to which both en and inv may contribute. Our results also indicate that, in the wing imaginal disc, en negatively regulates its own expression.

\section{MATERIALS AND METHODS}

\section{Fly stocks}

Wild-type flies were from the Oregon R strain (Lindsley and Zimm, 1992). GAL4 lines were as follows: MS 1096 from F. Jimenez (Capdevila and Guerrero, 1994), 71B, 30A (Brand and Perrimon, 1993), C-765,C-734, C-743, C-580 from A. Brand. The UAS- $h h$ line was described by Capdevila and Guerrero, (1994). The mutant stocks were as follows: $e n^{1}, e n^{2}$ are described by Lindsley and Zimm (1992); Lawrence and Morata (1976) and Morata et al. (1983). The dpp-lacZ (BS3.0, Blackman et al., 1991), en-lacZ (ry xho 25/CyO, Hama et al., 1990) and hh-lacZ (J413, Ma et al., 1993) stocks were kindly provided by W. Gelbart, T. Kornberg and K. Moses respectively.

\section{Generation of UAS-en transgenic fly lines}

For the production of the UAS-en transgenic fly lines, a EcoRI DNA fragment of $2 \mathrm{~kb}$ containing the entire en ORF was isolated from a plasmid (D2B) (Poole et al., 1985) and cloned into the EcoRI site of the pUAST plasmid. The recombinant plasmid containing the en cDNA in the correct orientation relative to the UAS sequences was used to transform Drosophila embryos from the stock $w^{118}$ by standard procedures of microinjection (Roberts, 1986). Several independent lines were obtained, all of them showing a similar level of gene expression as judged by immunostaining of imaginal discs using anti-en/inv monoclonal antibody that recognizes both en and inv antigens (Patel et al., 1989).

\section{Whole-mount immunostaining of imaginal discs}

Immunostainings using anti-ptc (Capdevila et al., 1994b), anti-en (Patel et al., 1989), anti-hh (Capdevila and Guerrero, 1994), and anti-ci (Slusarski et al., 1995) antisera were performed essentially as described by Capdevila et al. (1994a). The imaginal discs from wandering third instar larvae were fixed in 4\% paraformaldehyde in PBS for 20 minutes at room temperature and washed in PBS. For diaminobenzidine (DAB) staining, PBS containing $0.2 \%$ BSA, $0.1 \%$ saponin and $5 \%$ goat serum was used for blocking and for antibody incubation. Tissue was blocked for 1 hour and incubated overnight at $4^{\circ} \mathrm{C}$ in a dilution of the primary antibodie, washed and incubated in a 1/300 dilution of biotinylated antimouse (for en and ptc antibodies) or anti-rat (in the case of anti-hh and anti-ci antibodies) for 1 hour at room temperature. Discs were then washed in PBT (PBS containing 0.1\% Tween 20) and incubated for 30 minutes in Vector AB elite solution in PBT. After several washes in PBT, the reaction was developed in $0.5 \mathrm{mg} / \mathrm{ml}$ DAB (Sigma) in PBS containing $0.06 \% \mathrm{H}_{2} \mathrm{O}_{2}$. Discs were mounted under coverslips in eponaraldite (Fluka) after dehydration.

Imaginal discs were observed and photographed under a Zeiss Axiophot microscope.

\section{X-Gal staining}

Imaginal discs were first fixed in 4\% paraformaldehyde in PBS for 20 minutes at room temperature, fixed again in $0.5 \%$ glutaraldehyde (Fluka) in PBS on ice for 2 minutes and washed in PBS. The reaction was developed in $5 \mathrm{mM} \mathrm{K}_{4}\left[\mathrm{Fe}^{\mathrm{II}}(\mathrm{CN})_{6}\right], 5 \mathrm{mM} \mathrm{K}_{3}\left[\mathrm{Fe}^{\mathrm{III}}(\mathrm{CN})_{6}\right], 1 \mathrm{mM} \mathrm{MgCl}_{2}$ and $0.2 \% \mathrm{X}-\mathrm{Gal}$ in PBS containing $0.3 \%$ Triton X-100. Discs were mounted and observed as described for diaminobenzidine staining.

\section{Whole-mount RNA in situ hybridization}

An inv DNA fragment of 684bp (7-690 bp) from the first exon of the gene was obtained by PCR from genomic DNA using two synthetic oligonucleotides as primers (5'GGAAGCTTACCTTGGCCAGCACTCG 3', and 5' CCGGATCCCAGCTGAGCAAGTGCATC 3') and subcloned in the Bluescript vector. This DNA fragment does not hybridize to the en DNA sequence. Synthesis of both invand $e n$-specific probes was achieved by unidirectional PCR. The following synthetic oligonucleotides were used as primers: $5^{\prime}$ AGCAGTGCTGCTGAATGC 3' for the inv probe and 5' GCTCTAGAGCGTGGAAACTCATGTCAC 3' for the en probe. Standard PCR conditions and DIG-labeled nucleotides (Boehringer Mannheim) were used. In situ hybridization (Tautz and Pfeiffle, 1989) was done as previously described (Capdevila et al., 1994a).

\section{RESULTS}

\section{Ectopic engrailed expression leads to a transformation of anterior into posterior patterns in the wing}

We have obtained transformed flies carrying a construct in which an en cDNA is under UAS control (see Materials and Methods) in order to express the en product ectopically using the GAL4 system (Brand and Perrimon, 1993). As ectopic expression of en during embryogenesis is bound to 
be lethal, we used some GAL4 lines driving expression of the GAL4 protein restricted to portions of the imaginal discs, which allow survival to adulthood. Some of them have already been described (Brand and Perrimon, 1993; Capdevila and Guerrero, 1994). As they act in different regions of the wing disc and with different levels, positional and quantitative differences in en expression can be tested. We have crossed the UAS-en flies to the following GAL4 lines: MS 1096, (gift from F. Jimenez, see also Capdevila and Guerrero, 1994), 30A, 71B (Brand and Perrimon, 1993), C-765, C-580, C-734, C-743 (gifts from Andrea Brand) to direct the en product in the anterior and the posterior wing compartments.

In the MS 1096/UAS-en line there is en product all over the dorsal wing pouch (Fig. 1D), although there is also some expansion into the ventral part of the disc at the end of the third instar. As shown in Fig. 1E,F, the anterior dorsal wing develops a posterior pattern. Characteristic posterior pattern elements such as the double row hairs and the axillary cord appear in the anterior margin. We find that the transformed dorsal compartment is substantially smaller than the untransformed ventral one (Fig. 1E), suggesting that the control of size and proliferation is, at least to a certain degree, independent in dorsal and ventral wing compartments.

In the 71B line the GAL4 expression is localized in dorsal and ventral regions of the wing pouch but it does not extend to the dorsoventral border (Fig. 1G). The 71B/UAS-en flies present the vein pattern of part of the anterior compartment transformed into the corresponding posterior pattern (Fig. $1 \mathrm{H}, \mathrm{I})$, indicating that not only the margin, but also other regions of the compartment can be transformed by en. The $\mathrm{C}$ 734 line produces dorsal and ventral expression away from the dorsoventral border and also results in a similar transformation in the vein pattern. These results clearly argue for the instructive property of the en product being in the specification of posterior patterns.

\section{The transformation induced by engrailed is associated with a change in the expression of specific anterior and posterior patterning genes}

The homeotic transformations described above are accompanied by changes in the expression of genes that are normally transcribed specifically in the anterior or posterior compartments. The anteriorly expressed genes cubitus interruptus (ci), patched (ptc) and dpp, (whose expression patterns are shown in Fig. 2A,C,E) are repressed in the area of en expression, (Fig. $2 \mathrm{~B}, \mathrm{D}, \mathrm{F})$, although they become active in the surrounding region (see below). The posterior gene $h h$ (Fig. $2 \mathrm{~J}$ ) becomes activated in en-expressing cells. Moreover, using a specific probe for the posterior gene inv (see Material and Methods), we have been able to study inv expression in lines with ectopic en expression. In normal development inv protein has the same distribution as en in both embryos and imaginal discs (Coleman et al., 1987; Fig. 2K). We find that the presence of the en product in the anterior compartment of UAS-en/MS 1096 flies produces an ectopic expression of inv (Fig. 2L) in the same en-expressing cells. This indicates a regulatory interaction between $e n$ and inv.

\section{invected and engrailed are activated by hedgehog}

When ectopically expressing $h h$ in MS1096/UAS- $h h$ flies
(Fig. 3B), we have observed posterior transformation of the anterior margin (Fig. 3A) similar to that found in MS1096/UAS-en, suggesting derepression of en and/or inv. This is visualized by the staining with the monoclonal antibody mAb4D9 (Patel et al., 1989), which recognizes both products. The staining extends to the anterior compartment of MS1096/UAS- $h h$ wing discs (Fig. 3D). The possibility of a specific effect on either en or inv was checked by in situ hybridization using specific probes for each gene. The result is that both en (Fig. 3G) and inv (Fig. 3F) are derepressed. Surprisingly, we found no alteration of lacZ expression when using the en-lacZ line Xho25 (Hama et al., 1990) as a marker of $e n$ activity, indicating that this line does not respond to $h h$ as the endogenous gene. Additionally, we have also found (using the hh-lacZ line J413; Ma et al., 1993) that, as a consequence of $e n$ activation, the $h h$ endogenous gene is in turn activated.

The ectopic expression of $e n$ and $i n v$ as a response to $h h$ has also been found in other GAL4 lines (30A and C-765) which also exhibit posterior transformation in the anterior compartments. These transformations are sometimes difficult to detect, perhaps because $d p p$ and $p t c$, both induced by $h h$ (Fig. 3C), would counteract the posterior transformations induced by en, or because the levels of en induced by UAS- $h h$ are in some cases insufficient to produce a posterior transformation.

\section{Ectopic activation of engrailed also produces duplications of anterior wing patterns}

In addition to the transformations of anterior into posterior wing described above, in some lines we also find duplications of anterior wing patterns associated with these transformations. This is especially clear in those lines that drive ectopic expression in restricted regions of the dorsal and ventral compartments along the dorsoventral border. The best example is the 30A line where the en product is induced in the proximal regions of the posterior and anterior compartments, both in the dorsal and ventral sides (Fig. 4A). In 30A/UAS-en flies, the costa, located in the proximal anterior wing, is transformed into axillary cord, a region located in an homologous position along the proximal-distal axis of the posterior compartment, but this is often accompanied by a duplication of adjacent anterior patterns (Fig. 4D). Since wing duplications are often associated with ectopic expression of $d p p$ (Capdevila and Guerrero, 1994), we have checked for a possible alteration of $d p p$ in these wings. We find an area of high levels of dpp product just adjacent to the region of ectopic en expression (Fig. 4B). This activation of $d p p$ is associated with high levels of $p t c$ and $c i$ in the same region, as is shown in Fig. 2D,F, for the MS 1096 line. This recreates the situation along the anteroposterior border, and as in this position, the $d p p$ gene initiates the signaling mechanism which eventually leads to a duplication of part of the anterior pattern (Capdevila et al., 1994; Tabata and Kornberg, 1994; Basler and Struhl, 1994; Capdevila and Guerrero, 1994). An induction of $d p p$ is also found by GAL4driven expression of $h h$ (Capdevila and Guerrero, 1994), but in this case the number of $d p p$-expressing cells is greater, since $h h$ and $d p p$ can be coexpressed in the same cells (Fig. $3 \mathrm{~B}, \mathrm{C})$. This results in bigger duplications and outgrowths of the wing. 


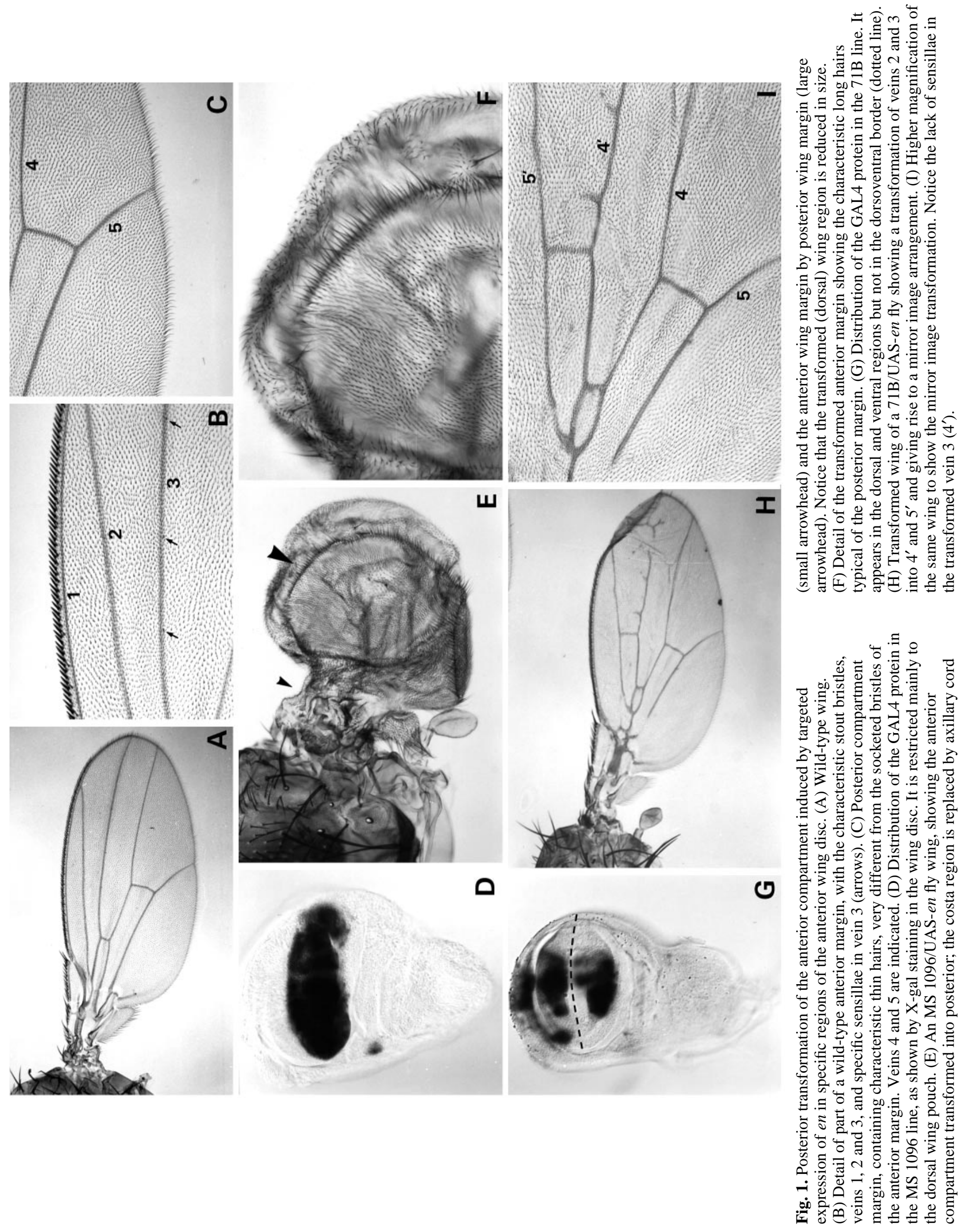




\section{Excess of engrailed function, but not of hedgehog, leads to a transformation of posterior into anterior patterns}

One surprising result found in most of the lines examined is that the excess of en product in the posterior compartment gives rise to a transformation towards anterior pattern. This leads to some paradoxical phenotypes like the one shown in Fig. 5D,E corresponding to an MS 1096/UAS-en wing, in
A
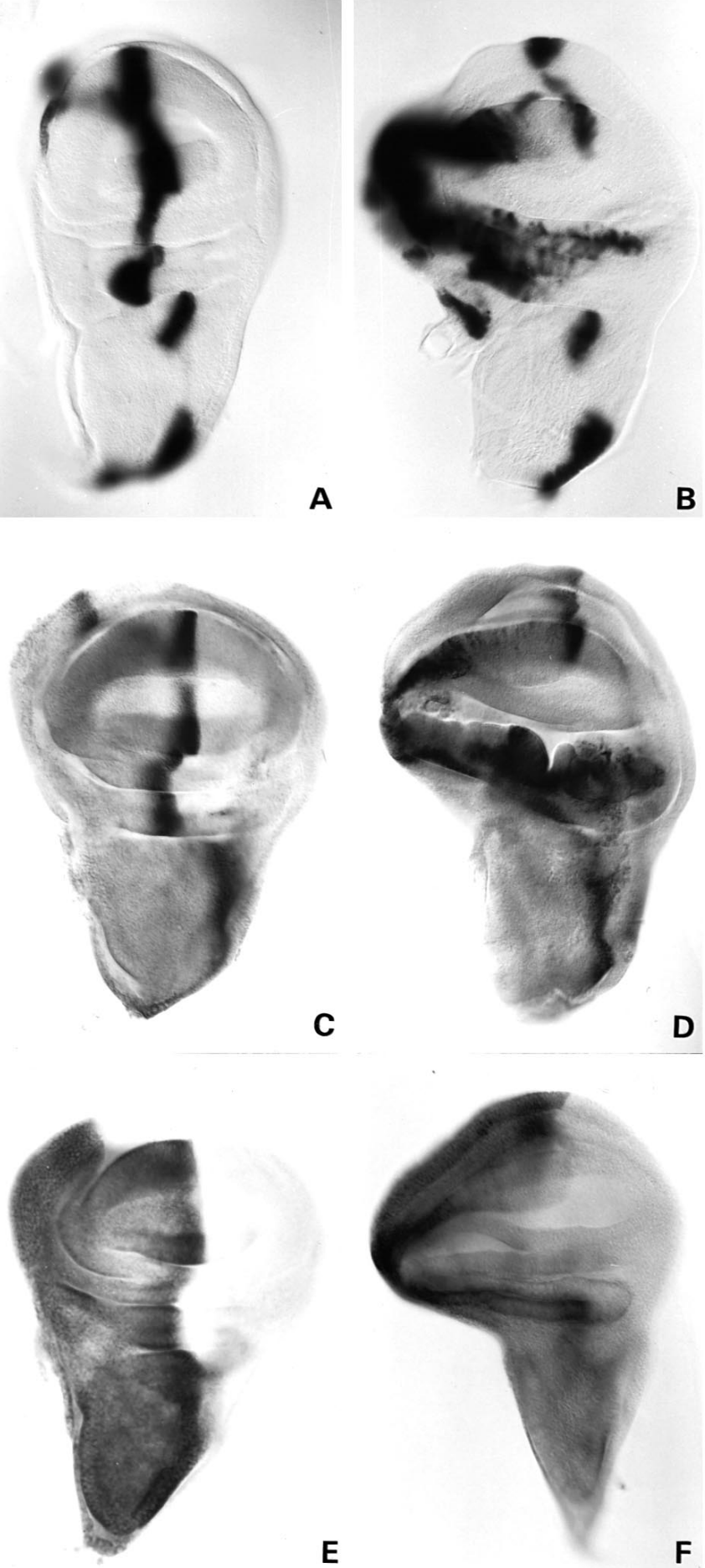

C

B

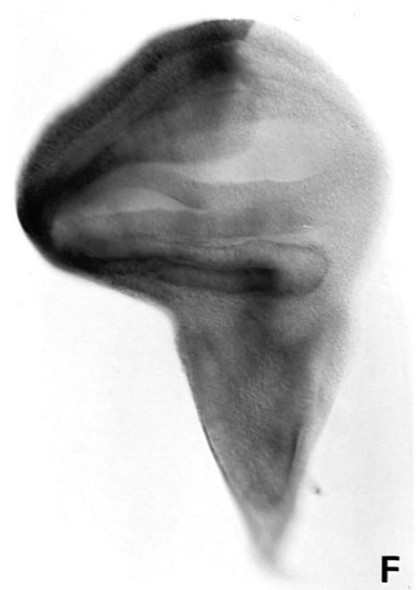

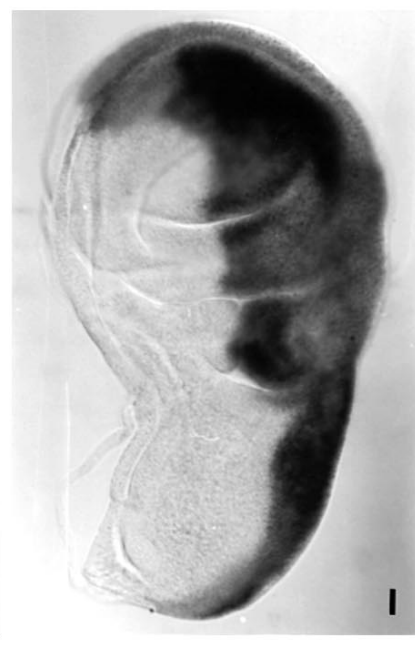

$\mathbf{G}$
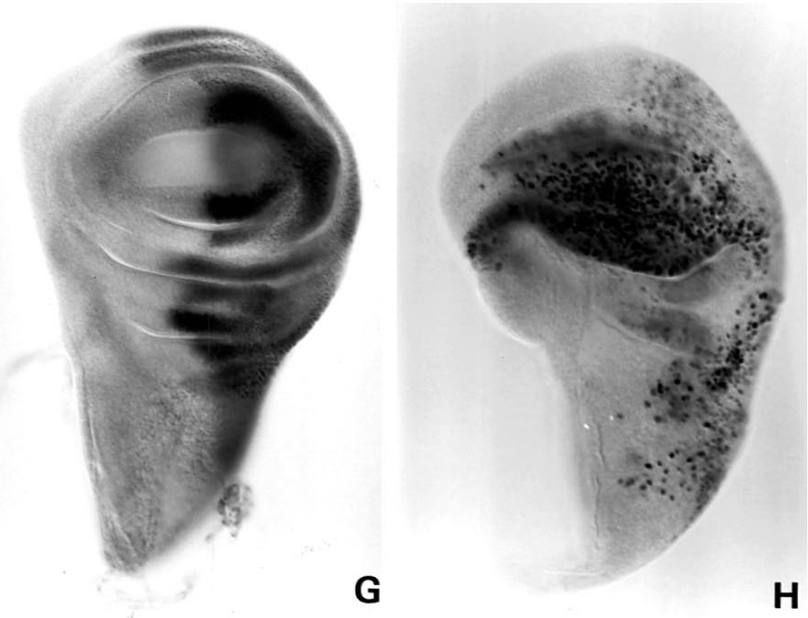

H

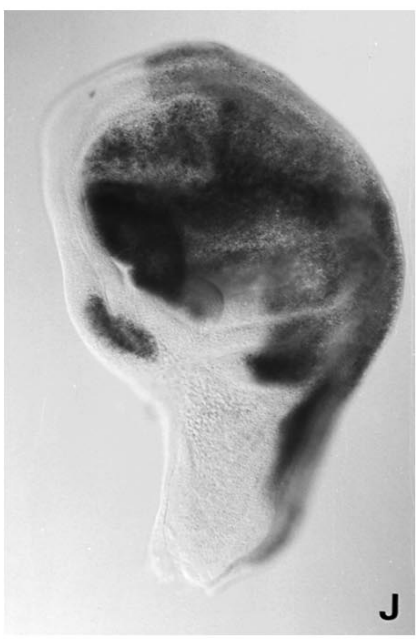

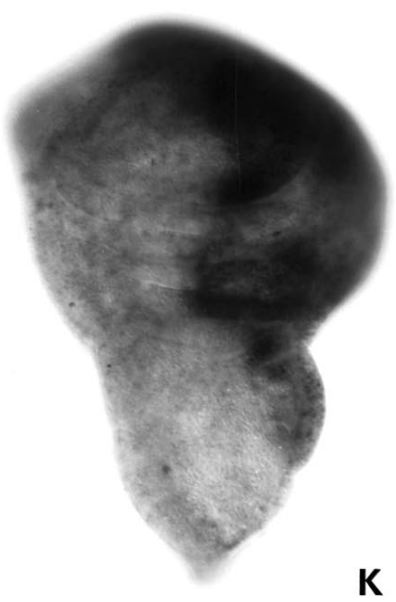

Fig. 2. Ectopic en activity modifies the expression pattern of anterior and posterior specific genes in the wing disc. (A,C,E) Wild-type expression of $d p p$ (X-gal staining), ptc (anti-ptc antibody staining) and $c i$ (anti-ci antibody staining) respectively. (B,D,F) Expression of the same genes in MS 1096/UAS-en flies. Notice the repression of the three genes in the area of en ectopic expression together with gain of function in the surrounding region. (G,I,K) Wild-type expression of en/inv (anti-en/inv antibody staining), $h h$ (X-gal staining) and inv (in situ hybridization) compared with (H,J,L) expression of the same genes in MS 1096/UAS-en flies. Note that $h h$ and inv expressions are induced in the same area as en. The arrowhead indicates a region in the posterior compartment showing loss of inv expression. 
which the anterior compartment is transformed into posterior, but the posterior one is transformed into anterior. This anterior transformation of the posterior compartment can occur anywhere along the proximodistal margin of the adult wings (Fig. 5B-E compare with Fig. 1C) and also in internal regions of the wing blade. In several instances the posterior compartments closely resemble those of $e n^{1} / e n^{1}$ or $e n^{1} / e n^{2}$ mutants (Fig. 5A).

Since the only difference between the anterior and the posterior compartments is the amount of en product, due to the summation in the posterior compartment of the endogenous plus the exogenous proteins, the result suggests that the excess of product is impairing the function of the endogenous en, and perhaps also inv, function. We have checked the expression of en and inv in MS 1096/UAS-en (Fig. 5G for en and Fig. 2L for $i n v)$ and in $e n^{1} / e n^{1}$ discs, and found that the levels of expression of the two genes are variably reduced in the posterior compartment. In addition, we have observed derepression of endogenous en gene in some cells of the anterior compartment (Fig. 5G). Occasionally (Fig. 4B), we also observe derepression of $d p p$ in the posterior compartment, probably due to the local loss of en (Raftery et al., 1991; Sanicola et al., 1995). However, we fail to see a reduction in $h h$ expression, which remains apparently normal. We do not know if the activation of the endogenous en gene is mediated by $h h$ or if it can occur by direct activation by the exogenous en product. The anterior transformation of the posterior compartment is not found in the MS 1096/UAS- $h$ h flies (compare Fig. 5D,E with Fig. 3A). This suggests that the high levels of en product are directly responsible for the reduction in inv expression in the posterior compartments of MS 1096/UAS-en flies.

The anterior transformation of the posterior compartment by excess of en product is hard to explain because the GAL4 driven en product should be sufficient to rescue the loss of the endogenous gene. In principle, one explanation could be that inv, which is also down regulated by the excess of en product,
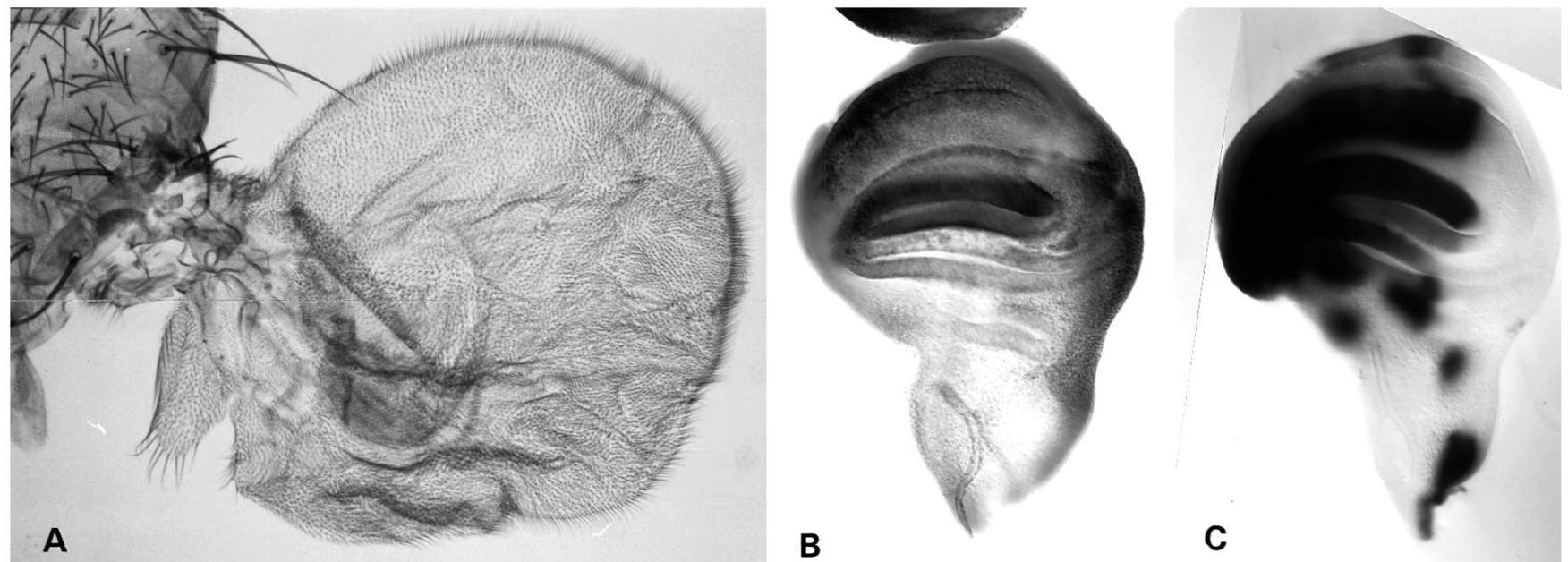

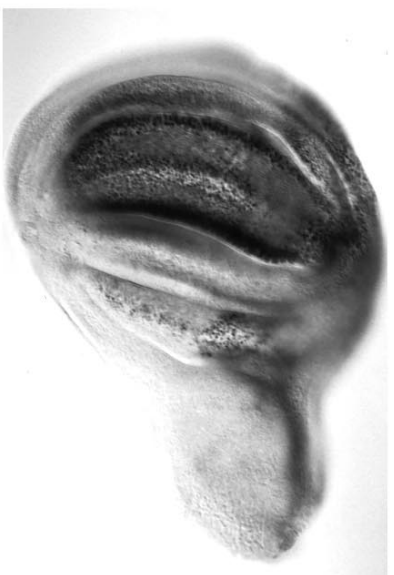

D

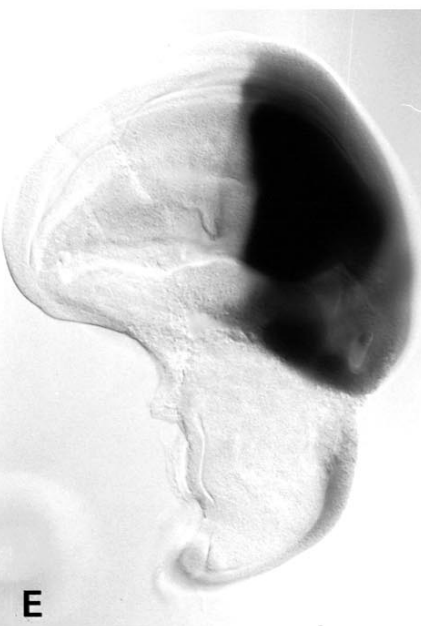

B

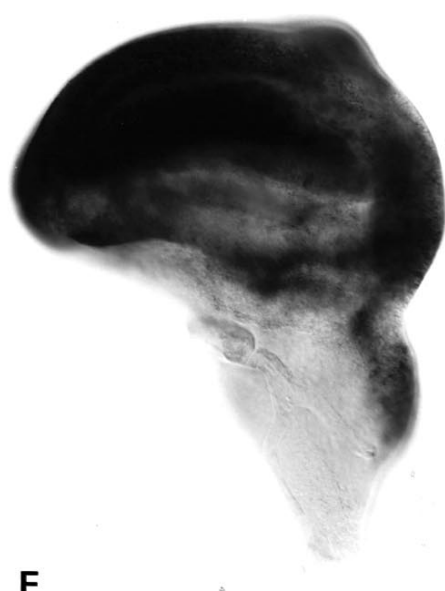

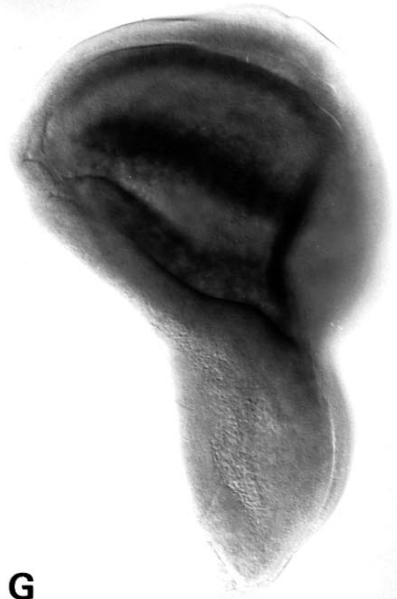

Fig. 3. Transformation of anterior to posterior wing by ectopic expression of $h h$ in the MS 1096/UAS- $h h$ genotype. (A) A wing exhibiting the posterior transformation clearly visible along the wing margin, now of posterior identity. (B) Distribution of the hh protein in the wing disc, where it extends to the anterior compartment. (C) $d p p$ expression as reported by $d p p$-lacZ (BS-3.0) line; $d p p$ is expressed in most of the cells in the anterior compartment that contain hh product. (D) Distribution of the en-inv antigen in a wing disc. The staining extends to the anterior compartment whereas normally it is restricted to the posterior compartment. (E) en expression as visualized by the en-lacZ line. $\beta$-gal distribution is confined to the posterior compartment. (F) inv expression as shown by the specific inv probe. (G) en expression as indicated by a specific en probe. Both en and inv are ectopically expressed in the anterior compartment and their expression patterns mimic that of $h h$ in the same line. 
has a major role in the posterior wing pattern which cannot be covered by the GAL4 driven en product.

\section{DISCUSSION}

\section{The function of engrailed in the wing}

In the experiments reported here we have used the GAL4 method to express ectopically the en product in anterior wing compartments. This produces a homeotic transformation of the region in the anterior wing where the en product is expressed in a posterior wing region located in a similar proximodistal position (see below). This clearly demonstrates the ability of the en product to specify adult posterior patterns and indicates that $e n$ is responsible for posterior adult identity in normal development (Morata and Lawrence, 1975).

The change in identity of the anterior cells is associated with changes in the expression patterns of genes specific for the anterior and posterior compartments. Anteriorly expressed genes, like $c i, p t c$ and $d p p$, are repressed in the area where $e n$ is ectopically expressed, while posterior genes such as $h h$ and inv are activated. Thus, one conclusion from our results is that inv is regulated by its closely linked gene en, as recently suggested by Goldsborough and Kornberg (1994). It is, however, unclear whether inv contributes to the posterior transformation. A possible role of inv is suggested by the results of Hidalgo (1994), who showed that posterior cell clones lacking both en and inv produce a stronger transformation than those lacking only en. The isolation of specific mutations in the inv locus may settle the issue about the specific role of inv.

In addition to the posterior transformations of anterior patterns discussed above, we often observe duplications of anterior wing patterns just adjacent to the regions exhibiting posterior transformations. Analysis of gene expression in the vicinity of the en-expressing region shows that $d p p$ is ectopically activated just outside this region. This is also accompanied by high local levels of ptc and ci proteins, very much resembling the situation in the anteroposterior boundary. The ectopic activation of $h h$ in the area of en ectopic expression generates an interface of $h h$ - and non- $h h$-expressing cells which results in local activation of $d p p$ as a consequence of blocking the repressive effect of $p t c$ upon $d p p$ expression. This produces a transformation similar to that achieved by direct ectopic expression of $d p p$ (Capdevila and Guerrero, 1994). Pattern duplications are only found in those GAL4 lines inducing local areas of $d p p$ transcription within the disc. It is possible that a graded distribution of dpp product is a requisite for the generation of wing patterns.

Altogether, our experiments suggest that the overall function of $e n$ in wing disc development can be separated into two distinct roles.

The first one is the primordial function in the cells of the posterior compartment to specify the characteristic posterior pathway (Morata and Lawrence, 1975; Lawrence and Morata, 1976). This is presumably achieved through the activation of posterior genes (and/or the repression of the anterior ones), although the mechanism is not known. The second role is indirect and connected with the overall specification of pattern in the appendage. It is achieved by the interface between cells of the posterior compartment expressing hh product (as activated by en) with anterior cells not transcribing it. The hh product is passed to the adjacent anterior cells (Tabata and Kornberg, 1994; Basler and Struhl, 1994; Capdevila and Guerrero, 1994), which respond by activating $d p p$ (Capdevila and Guerrero, 1994). The

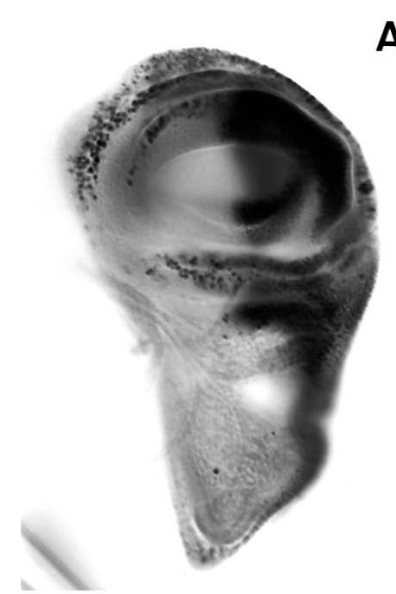

A
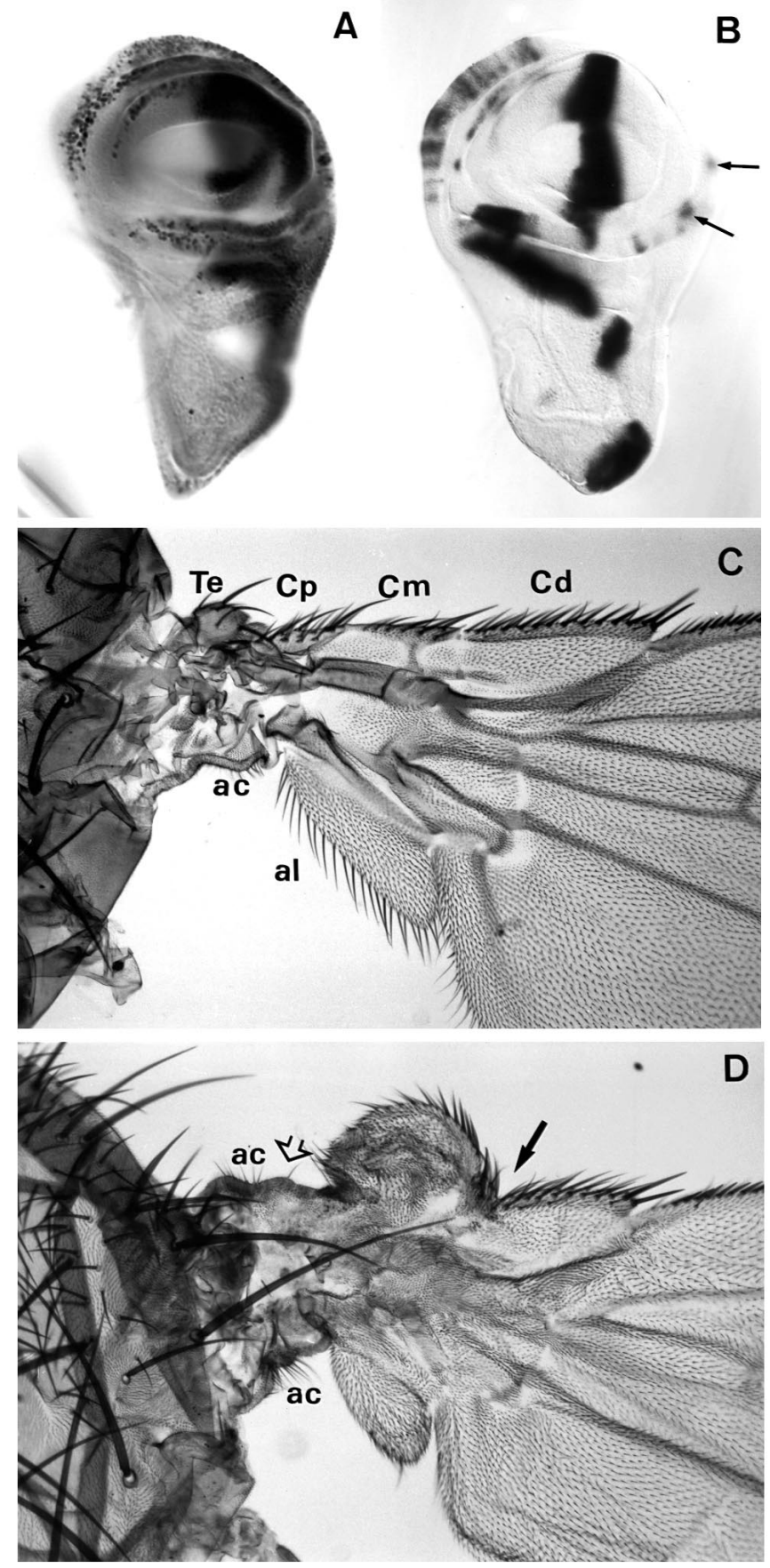

Fig. 4. Effect of ectopic en expression in 30A/UAS-en flies.

(A) Distribution of en and inv proteins in a 30A/UAS-en wing imaginal disc. (B) Ectopic $d p p$ expression in a disc of the same genotype. The arrows indicates $d p p$ derepression in the posterior compartment. (C) Proximal part of a wild-type wing. Note the tegula $(\mathrm{Te})$, and proximal $(\mathrm{Cp})$, medial $(\mathrm{Cm})$ and distal $(\mathrm{Cd})$ costa, in the anterior compartment, and axillary cord (ac) and alula (al) in the posterior compartment. (D) Mirror image duplication in the anterior compartment of a 30A/UAS-en wing, showing the replacement of $\mathrm{Te}, \mathrm{Cp}$ and $\mathrm{Cm}$ by ac (open arrow) and the duplication axis of the $\mathrm{Cd}$ distal pattern (arrow). 
expression of $d p p$ restricted to the anteroposterior boundary has to be precisely controlled and is achieved through the function of ptc, $c i$ and other genes (Capdevila and Guerrero, 1994). From this region the morphogenetic influence of $d p p$ spreads to anterior and posterior compartment cells. In the absence of any other modifying factor, the two wing compartments would develop anterior patterns in mirror image symmetry (e.g. as in an $e n^{1}$ homo-zygous wing). These two distinct roles of $e n$ can be clearly visualized in the $30 \mathrm{~A} / \mathrm{UAS}$-en flies in which the en product is planted in the proximal anterior wing region: the proximal costa is transformed into an axillary cord, a characteristic posterior structure, and in addition, there is a duplication of anterior wing patterns (Fig. 4). Changes in both patterns are caused by the presence of the en product.

\section{The activation of en and inv by $h h$}

The activation of $e n$ and inv by $h h$ observed in several GAL4 lines $(1096,30 \mathrm{~A}, \mathrm{C}-765)$ is an unexpected result, the functional significance of which is unclear, but that could be responsible for the late activation of en observed in some cells of the anterior compartment in third instar wing imaginal discs (Blair, 1992). This late anterior en/inv expression may have a patterning function in the region between vein 3 and the compartment border (Hidalgo, 1994). The activation of en/inv by $h h$ could be achieved by an unknown mechanism or perhaps a consequence of blocking the repressive effect of $p t c$, as occurs for $d p p$ and ptc itself (Capdevila et al., 1994a; Tabata and Kornberg, 1994; Basler and Struhl, 1994; Capdevila and Guerrero, 1994).

Although the activation of en and inv by $h h$ has only been
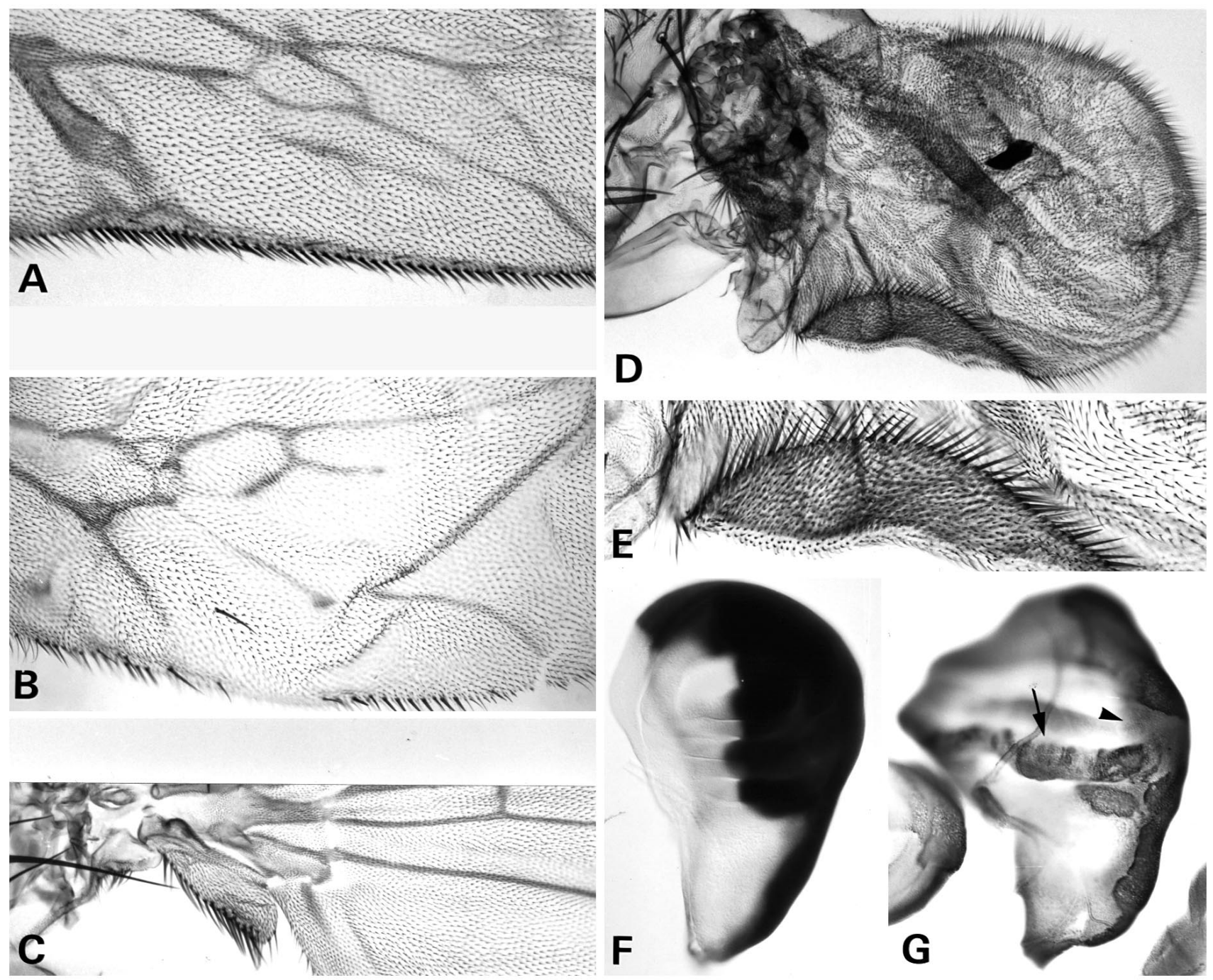

Fig. 5. Anterior transformation of the posterior compartment induced by high levels of en product. (A) Part of the posterior compartment of an $e n^{1} / e n^{2}$ wing showing anterior transformation, especially visible by the appearance of the stout bristles characteristic of the anterior margin (see Fig. 1B). (B) Posterior margin of a C-743/UAS-en wing displaying a similar transformation. (C) Proximal part of a 30A/UAS-en wing showing socketed costa-like bristles in the alula region, also indicating an anterior transformation. (D) Transformed wing of an MS 1096/UAS-en fly. (E) Detail of the transformed posterior margin showing the appearance of stout bristles all over the edge. (F) Normal en expression as monitored by X-gal staining in the en-lacZ line in an untransformed disc. (G) en expression in an MS 1096/UAS-en wing disc, also carrying the en-lacZ gene and developed using anti- $\beta$-gal antibody staining. It shows a reduction in the amount of $\beta$-gal in the dorsal posterior region of the wing pouch (arrowhead), precisely the region where GAL4-induced en product adds to the endogenous product. A derepression of en endogenous gene is also found in some cells of the anterior compartment (arrow). 
observed in the anterior compartment, it is possible that it also occurs in the posterior compartment, where it might have a role, connected, for example, with the maintenance of en activity. However, all identified elements of the $h h$ signal involved in $d p p$ activation in the wing disc, like $p t c$ or protein kinase A (Capdevila et al., 1994; Jiang and Struhl, 1995; Li et al., 1995; Pan and Rubin, 1995; Lepage et al., 1995) have anterior compartment activity. Therefore, the possible activation of en/inv by $h h$ in the posterior compartment may suggest the existence of an unidentified receptor and/or a different transduction pathway specific to the posterior compartment.

\section{Excess engrailed product causes loss of function engrailed phenotypes}

The excess of en product in the posterior compartment of some GAL4/UAS-en discs, results in phenotypes similar to those of mutations like $e n^{1}$ or $e n^{2}$ (Fig. 5A), known to cause partial loss of en function (Brower, 1986; Raftery et al., 1991; our own observations). As in the case of $e n^{1}$ mutants, we also observe a loss of the endogenous en and inv gene products in GAL4/UAS-en discs. Here, there is a situation in which normal levels of en activate inv, but inappropriate high levels produce the opposite effect, perhaps by direct repression on en and inv transcription. It has recently been suggested (Goldsborough and Kornberg, 1994) that en and inv share regulatory elements, which may explain why the two genes are repressed by the excess of en function. Apparently, this repression does not involve $h h$, for we do not see a reduction in $h h$ expression in the posterior compartments of MS 1096/UAS-en flies. Moreover, the excess of hh product in the posterior compartments of MS 1096/UAS- $h h$ flies does not affect the posterior pattern, also arguing against an involvement of $h h$.

The functional significance of this phenomenon is uncertain at present; it obviously indicates that the amount of en product has to be precisely regulated and also provides an explanation for the observation that heat-shock-induced high levels of en product in embryogenesis produce a phenotype resembling that of $e^{-}$embryos (Poole and Kornberg, 1988). There are some cases reported in which different levels of the regulatory product may also have opposite effects; the Antp product upregulates $\mathrm{Scr}$ at moderate levels but suppresses its expression at high levels (Pelaz et al., 1993).

We are very grateful to A. Brand, F. Jimenez, C. Parras, R. Holmgren, J. Kassis, T. Kornberg, W. Gelbart, P. Beachy, K. Moses and N. Perrimon for providing stocks, antibodies and plasmids. We thank R. González for technical assistance. We want to thank J. F. de Celis and M. Ruiz-Gomez for communicating unpublished results. We are supported by grants from the Dirección General de Investigación Científica y Técnica, the International Human Frontier Science Program (372/94 M) and an institutional grant from the Ramon Areces Foundation. Isabel Guillen was recipient of a fellowship by the Instituto de Cooperación Iberoamericana.

\section{REFERENCES}

Basler, K. and Struhl, G. (1994). Compartment boundaries and the control of Drosophila limb pattern by hedgehog protein. Nature 368, 208-214.

Blackman, R. K., Sanicola, M., Raftery, L. A., Gillevet, T. and Gelbart, W. M. (1991) An extensive 3' cis-regulatory region directs the imaginal disc expression of decapentaplegic, a member of a TGF- $\beta$ family in Drosophila. Development 111, 657-665
Blair, S. S. (1992). engrailed expression in the anterior lineage compartment of the developing wing blade of Drosophila. Development 115, 21-33.

Brand, A. and Perrimon, N. (1993). Targeted gene expression as a means of altering cell fates and generating dominant phenotypes. Development 118, 401-415.

Brower, D. (1986). engrailed gene expression in Drosophila imaginal discs. EMBO J. 5, 2649-2656.

Capdevila, J., Estrada, M. P., Sánchez-Herrero, E. and Guerrero, I. (1994a). The Drosophila segment polarity gene patched interacts with decapentaplegic in wing development. EMBO J. 13,71-82

Capdevila, J., Pariente, F., Sampedro, J., Alonso, J. L., and Guerrero, I. (1994b). Subcellular localization of the segment polarity protein patched suggests an interaction with the wingless reception complex in Drosophila embryos. Development 120, 987-998.

Capdevila, J. and Guerrero, I. (1994). Targeted expression of the signaling molecule decapentaplegic induces pattern duplications and growth alterations in Drosophila wings. EMBO J. 13, 4459-4468.

Coleman, K. G., Poole, S. J., Weir, M. P., Soller, W. C., and Kornberg, T. B. (1987). The invected gene of Drosophila: sequence analysis and expression studies reveal a close kinship to the engrailed gene. Genes Dev. 1, 18-28.

DiNardo, S., Kuner, J. M., Theis, J. and O'Farrell, P. H. (1985). Development of embryonic pattern in Drosophila melanogaster as revealed by accumulation of the nuclear engrailed protein. Cell 43, 59-69.

Fjose, A., McGinnis, W. J. and Gehring, W. J. (1985). Isolation of a homeobox-containing gene from the engrailed region of Drosophila and the spatial distribution of its transcripts. Nature 313, 284-289.

García-Bellido, A. and Santamaría, P. (1972). Developmental analysis of the wing disc in the mutant engrailed of Drosophila melanogaster. Genetics 72, 87-104

García-Bellido, A., Ripoll, P. and Morata, G. (1973). Developmental compartmentalization of the wing disk of Drosophila. Nature 245, 251-253.

Goldsborough, A. S. and Kornberg, T. K. (1994). Allele-specific quantification of Drosophila and engrailed transcripts. Proc. Natl. Acad. Sci. USA 91, 12696-12700.

Gubb, D. (1985). Further studies on engrailed mutants in Drosophila melanogaster. Roux's Arch. Dev. Biol. 194, 236-246.

Hama, C., Ali, Z. and Kornberg, T. B. (1990). Region-specific recombination and expression are directed by portions of the Drosophila engrailed promoter. Genes Dev. 4, 1079-1093.

Hidalgo, A. (1994). Three distinct roles for the engrailed gene in Drosophila wing development. Curr. Biol. 1, 1087-1098.

Ingham, P. W., Martínez Arias, A. Lawrence, P. A. and Howard, K. (1985). Expression of engrailed in the parasegment of Drosophila. Nature 317, 634636.

Jiang, J. and Struhl, G. (1995). Protein kinase A and hedgehog signaling in Drosophila limb development. Cell 80, 563-572.

Kornberg, T. B. (1981a). engrailed: a gene controlling compartment and segment formation in Drosophila. Proc. Natl. Acad. Sci. USA 78, 1095-1099.

Kornberg, T. B. (1981b). Compartments in the abdomen of Drosophila and the role of engrailed locus. Dev. Biol. 86, 363-372.

Kornberg, T. B., Siden, I., O'Farrell, P., and Simon, M. (1985). The engrailed locus of Drosophila: In situ localization of transcripts reveals compartment specific expression. Cell 40, 45-53.

Lawrence, P. A. (1992). The Making of a Fly. Oxford: Blackwell Scientific Publications.

Lawrence, P. A. and Morata, G. (1976). Compartments in the wing of Drosophila: a study of the engrailed gene. Dev. Biol. 50, 321-337.

Lawrence, P. A. and Struhl, G. (1982). Further studies of the engrailed phenotype in Drosophila. EMBO J. 1, 827-833.

Lee, J., Kessler, D., Parks, S. and Beachy, P. (1992). Secretion and localized transcription suggest a role in positional signaling for products of the segmentation gene hedgehog. Cell 71, 33-50.

Li, W., Ohlmeyer, J. T., Lane, M. E. and Kalderon, D. (1995). Function of protein kinase A in hedgehog signal transduction and Drosophila imaginal disc development. Cell 80, 553-562.

LePage, T., Cohen, S., Díaz-Benjumea, F. J. and Parkhurst, S. M. (1995). Signal transduction by cAMP-dependent protein kinase A in Drosophila limb patterning. Nature 373, 711-715.

Lindsley, D. L. and Zimm, G. G. (1992). The Genome of Drosophila melanogaster. San Diego: Academic Press, Inc.

Ma, C., Zhou, Y., Beachy, P. A. and Moses, K. (1993). The segment polarity gene hedgehog is required for progression of the morphogenetic furrow in the development Drosophila eye. Cell 75, 927-938. 
Morata, G. and Lawrence, P. A. (1975). Control of compartment development by the engrailed gene in Drosophila. Nature 255, 614-617.

Morata, G. and Lawrence, P. A. (1979). The development of the eye-antennal disc of Drosophila. Dev. Biol. 70, 355-371.

Morata, G., Kornberg, T. and Lawrence, P. A. (1983). The phenotype of engrailed mutations in the antenna of Drosophila. Dev. Biol. 99, 27-33.

Pan, D. and Rubin, G. M. (1995). cAMP-dependent protein kinase A in hedgehog signal transduction and Drosophila imaginal disc development. Cell 80, 543-552.

Patel, N. H., Martín-Blanco, E., Coleman, K. G., Poole, S. P., Ellis, M. C., Kornberg, T. B. and Goodman, C. S. (1989). Expression of engrailed proteins in arthropods, annelids, and chordates. Cell 58, 955-968.

Pelaz, S. Urquia, N. and Morata, G. (1993). Normal and ectopic domains of the homeotic gene Sex combs reduced of Drosophila. Development 117, 917-923.

Poole, S. J. and Kornberg, T. K. (1988). Modifying expression of the engrailed gene of Drosophila melanogaster. Development 104 (Supl), 85-94

Poole, S. J., Kauvar, L. M., Drees, B. and Kornberg, T. (1985). The engrailed locus of Drosophila: structural analysis of an embryonic transcript. Cell 40, 37-43.

Raftery, L. A., Sanicola, M., Blackman, R. K., and Gelbart,W. M. (1991).
The relationship of decapentaplegic and engrailed expression in Drosophila imaginal discs: do these genes mark the anteroposterior compartment boundary? Development 113, 27-33.

Roberts, D. B. . (1986). Drosophila: A Practical Approach. Oxford: IRL Press. Sanicola, M. Sekelsky, J. Elson, S. and Gelbart, W. M. (1995). Drawing a stripe in Drosophila imaginal disks: negative regulation of decapentaplegic and patched expression by engrailed. Genetics 139, 754-756.

Slusarski, D. C. Motzny, C. K. and Holmgren, R. (1995). Mutations that alter the timing and pattern of cubitus interruptus gene expression in Drosophila melanogaster. Genetics 139, 229-240.

Tabata, T. and Kornberg, T. B. (1994). hedgehog is a signaling protein with a key role in patterning Drosophila imaginal discs. Cell 76, 89-102.

Tabata, T., Eaton, S. and Kornberg, T. B. (1992). The Drosophila hedgehog gene is expressed specifically in posterior compartment cells and it is a target of engrailed regulation. Genes Dev. 6, 2635-2645.

Tautz, D. and Pfeiffle, C. (1989). A non-radioactive in situ hybridization method for the localization of specific RNAs in Drosophila embryos reveals translational control of the segmentation gene hunchback. Chromosoma $\mathbf{9 8}$, $81-85$.

(Accepted 28 June 1995) 